\title{
Factor Analysis of Intraoperative Radical Conversion During Partial Nephrectomy - Single Surgeon Experience
}

\author{
Dong Hwan Lee ${ }^{1}$, Woo Heon Cha ${ }^{2}$, Younsoo Chung ${ }^{1}$, Tae Jin Kim ${ }^{1}$, In Jae Lee ${ }^{1}$, \\ Byeong Do Song ${ }^{1}$, Sangchul Lee ${ }^{3}$, Sung Kyu Hong ${ }^{3}$, Jong Jin $\mathrm{Oh}^{3}$, Seok-Soo Byun ${ }^{3}$ \\ ${ }^{1}$ Department of Urology, Seoul National University Bundang Hospital, Seongnam, Korea \\ ${ }^{2}$ Department of Urology, Gimcheon Medical Center, Gimcheon, Korea \\ ${ }^{3}$ Department of Urology, Seoul National University Bundang Hospital, Seoul National University College of \\ Medicine, Seongnam, Korea
}

\begin{abstract}
Purpose: Preoperative aspects and dimensions used for an anatomical (PADUA) classification is useful to predict perioperative complications and warm ischemia time. However, it remains uncertain whether PADUA classification can predict intraoperative conversion from partial nephrectomy (PN) to radical nephrectomy (RN). We evaluate whether PADUA classification parameters can predict conversion to RN during PN.

Materials and Methods: We retrospectively assessed data of 593 patients who underwent open PN and robotic PN for renal tumors by a single surgeon at a single tertiary center between December 2003 and September 2017. Intraoperative conversion to RN was performed in 17 of 593 patients who initially underwent PN. We evaluated the factors influencing the surgical modalities including PN and radical conversion cases. Then, the factors significantly associated with conversion to RN were further analyzed.

Results: There were no significant differences between the 2 groups in age, longitudinal location, exophytic rate, and rim or face locations of renal masses. Renal sinus involvement, urinary collecting system involvement, and renal mass size were variables that predicted radical conversion, using univariate analysis. Renal sinus involvement (odds ratio [OR], 9.075; $p=0.049$ ) and urinary collecting system involvement $(\mathrm{OR}, 3.944 ; p=0.029)$ were independent predictors of intraoperative radical conversion, using multivariate analysis.

Conclusions: The PADUA classification is a useful tool to predict intraoperative conversion from PN to RN. Renal sinus involvement and urinary collecting system involvement are the best predictors of intraoperative conversion from PN to RN. (Korean J Urol Oncol 2017;15:165-171)
\end{abstract}

Key Words: Nephrectomy $\cdot$ Operative surgical procedure $\cdot$ Classification $\cdot$ Prediction

Received November 1, 2017, Revised November 19, 2017,

Accepted November 21, 2017

Corresponding Author: Seok-Soo Byun

Department of Urology, Seoul National University Bundang Hospital, Seoul National University College of Medicine, 82 Gumi-ro 173beon-gil, Bundang-gu, Seongnam 13620, Korea

E-mail: ssbyun@snubh.org

Tel: +82-31-787-7342, Fax: +82-31-780-4057

ORCID code: https://orcid.org/0000-0001-9356-9500

\section{INTRODUCTION}

Partial nephrectomy (PN) is the gold-standard treatment of small renal masses, and shows similar results as radical nephrectomy (RN) in oncological outcomes. ${ }^{1} \mathrm{RN}$ causes renal insufficiency and cardiovascular events that lead to increased morbidity and mortality. ${ }^{2,3} \mathrm{PN}$ can provide excellent results in patients presenting with a renal mass in a solitary kidney, stage IV chronic kidney disease, multiple renal tumors, and hereditary

(i) (2) This is an Open Access article distributed under the terms of the Creative Commons Attribution Non-Commercial License (http://creativecommons.org/licenses/by-nc/4.0/) which permits unrestricted non-commercial use, distribution, and reproduction in any medium, provided the original work is properly cited. 2017 (C) Copyright The Korean Urological Oncology Society and The Korean Prostate Society. All Rights Reserved. 
disorder, thereby preventing the need for dialysis. ${ }^{4,5}$ Therefore PN should receive further attention as the treatment-of-choice for renal masses. ${ }^{6}$

Laparoscopic PN (LPN) and robotic PN (RPN) were introduced as minimally-invasive $\mathrm{PN}$ treatments. Hurber et al. reported that the results of RPN are similar to those obtained with LPN. $^{7}$ Long et al. ${ }^{7}$ reported that RPN had the merits of high definition, 3-dimensional optics, and wristed instruments. However open PN (OPN) was still preferred in more complex surgical cases. The radius exophytic/endophytic nearness anterior/posterior location (RENAL) system, Centrality-index (C-index), and preoperative aspects and dimensions used for an anatomical (PADUA) classification system are well-known assessment tools of renal masses, to determine whether a given renal tumor was a suitable candidate for PN. These have validity as assessment tools for renal tumors undergoing nephron-sparing surgery (NSS). The RENAL system was developed in 2009, and considers renal tumor size, exophytic extension, distance between the renal tumor and the renal sinus and urinary collecting system (UCS), and tumor location. ${ }^{8}$ The RENAL system has the advantage that the parameter measurements are generally easily measured. The exception is that it is not always easy to measure the distance between the tumor mass and the renal sinus/UCS, resulting in different distance estimates among observers. Also, coronal abdominopelvic computed tomography (CT) imaging or magnetic resonance imaging (MRI) is necessary to know the correlation between renal tumor orientation and renal polarity. ${ }^{9}$

The C-index was developed by Simmon in 2010, and calculates tumor size and renal site using preoperative contrast-enhanced CT imaging. This approach provided a better description of the renal tumor and is related to the postoperative nadir estimated glomerular filtration rate (eGFR) and the percent decrease in eGFR following PN. ${ }^{10}$ However, the C-index is not simple and did not precisely calculate the actual renal tumor location. Therefore, Long et al. ${ }^{7}$ said that the C-index did not predict PN perioperative outcome better than the PADUA and RENAL score systems.

The PADUA classification system is a tool for predicting patient outcome after NSS. This system was invented by urologists that were blinded to patient information. ${ }^{9}$ The PADUA system differs from the RENAL system primarily in its evaluation of renal sinus/UCS involvement of the renal tumor. Coronal and/or axial CT or MRI views are used to measure the renal sinus line. Reproducibility is excellent because measuring the renal tumor placement relative to the renal sinus/UCS is easier. This classification was useful for predicting postoperative complications, warm ischemia time, and estimated blood loss in PN. ${ }^{11-14}$ We did not locate any published reports regarding the use of the PADUA classification system in predicting intraoperative $\mathrm{RN}$ conversion in PN patients. Thus, we analyzed the reasons for intraoperative $\mathrm{PN}$ to $\mathrm{RN}$ conversion, and investigated the utility of the PADUA classification in predicting PN conversion to $\mathrm{RN}$.

\section{MATERIALS AND METHODS}

\section{Study Population}

Using the prospective continuous fill-up nephrectomy data base, patients with a renal tumor diagnosed through preoperative abdominopelvic CT or MRI that underwent OPN or RPN between December 2003 and September 2017 by a single surgeon at Seoul National University Bundang Hospital were evaluated for study participation. A total 593 patients were classified into 2 groups: Group 1 is the $\mathrm{PN}$ to $\mathrm{RN}$ conversion group $(\mathrm{n}=17)$, and group 2 is the PN group ( $\mathrm{n}=576)$. Written informed consent was waived by the board. This study was approved by the Institutional Review Board at the Seoul National University Bundang Hospital (approval number: B-1211-180-104).

\section{Surgical Outcome}

The 17 patients in group 1 required intraoperative conversion to $\mathrm{RN}$, and the 576 patients in group 2 received $\mathrm{PN}$ as originally scheduled. Patient age, sex, BMI, preoperative creatinine, preoperative eGFR (calculated using the Modification of Diet in Renal Disease study equation), EBL, renal mass assessment through abdominopelvic CT or MRI were compared between both groups.

\section{Tumor Assessment and Analysis of the Reasons for Intraoperative PN-RN Conversion}

We assessed renal tumors using the PADUA classification system. Tumor assessment was composed of 2 steps. After 4 senior residents and 3 urology fellows received detailed instruction for renal tumor assessment, they evaluated the PADUA score of each patient. These initial assessment data were then reviewed by another fellow urologist. All investigators were blinded to patient outcomes. Multiple potential 
reasons for intraoperative PN conversion to RN were analyzed, 7 including each item of PADUA system, to determine the predictive power of these parameters for estimating conversion to RN in OPN and RPN patients.

\section{Statistical Analysis}

Statistical analysis was performed by using IBM SPSS Statistics ver. 22.0 (IBM Co., Armonk, NY, USA). Continuous variables between the 2 groups were tested using Student t-test. Nominal parameters were analyzed using Pearson chi-square and Fischer exact test. Predictors of conversion to RN were assessed through univariate and multivariate logistic regression. A p-value less than 0.05 was considered indicative of a statistically significant difference.

\section{RESULTS}

\section{Demographic and Clinical Data}

The mean age was $54.9 \pm 11.8$ years for group $1(\mathrm{n}=17)$ and $53.4 \pm 12.8$ years for group $2(\mathrm{n}=576)$. Male sex was 15 persons (88.2\%) for group 1, and 400 persons $(69.4 \%)$ for group 2. There were no statistical differences between groups for preoperative creatinine or preoperative eGFR. The EBL was $348.6 \pm 336.3 \mathrm{~mL}$ for group 1 and $195.3 \pm 156.5 \mathrm{~mL}$ for group $2(\mathrm{p}<0.001)$. Tumor size was $5.2 \pm 2.1 \mathrm{~cm}$ for group 1 and $3.2 \pm 1.8 \mathrm{~cm}$ for group $2(\mathrm{p}<0.001)$. The total PADUA classification scores were $10.0 \pm 1.7$ for group 1 and $8.4 \pm 1.5$ for group $2(\mathrm{p}<0.001)$ (Table 1$)$.

\section{Factors Influencing Intraoperative PN Conversion to RN}

Four cases were converted from open $\mathrm{PN}$ to open $\mathrm{RN}$, and

Table 1. Demographic and clinical data

\begin{tabular}{|c|c|c|c|}
\hline Variable & Group $1(n=17)$ & Group $2(n=576)$ & p-value \\
\hline Age (yr) & $54.9 \pm 11.8$ & $53.4 \pm 12.8$ & 0.620 \\
\hline Sex & & & 0.096 \\
\hline Male & $15(88.2)$ & $400(69.4)$ & \\
\hline Female & $2(11.8)$ & $176(30.6)$ & \\
\hline Site & & & 0.076 \\
\hline Right & $12(70.6)$ & $281(48.8)$ & \\
\hline Left & $5(29.4)$ & $295(51.2)$ & \\
\hline Body mass index $\left(\mathrm{kg} / \mathrm{m}^{2}\right)$ & $25.2 \pm 2.9$ & $25.1 \pm 3.6$ & 0.933 \\
\hline Preoperative creatinine & $1.0 \pm 0.2$ & $0.9 \pm 0.5$ & 0.648 \\
\hline Preoperative eGFR & $87.8 \pm 19.5$ & $91.3 \pm 18.7$ & 0.455 \\
\hline Operation time (min) & $162.7 \pm 62.1$ & $148.3 \pm 66.9$ & 0.426 \\
\hline $\mathrm{EBL}(\mathrm{mL})$ & $348.6 \pm 336.3$ & $195.3 \pm 156.5$ & 0.001 \\
\hline Clinical size $(\mathrm{cm})$ & $5.2 \pm 2.1$ & $3.2 \pm 1.8$ & $<0.001$ \\
\hline Longitudinal location (\%) & & & 0.856 \\
\hline Superior & $6(35.3)$ & $198(34.4)$ & \\
\hline Middle & $6(35.3)$ & $226(39.2)$ & \\
\hline Inferior & $5(29.4)$ & $152(26.4)$ & \\
\hline Tumor size $(\mathrm{cm})$ & & & $<0.001$ \\
\hline$<4$ & $6(35.3)$ & $449(78.0)$ & \\
\hline $4-7$ & $7(41.2)$ & $111(19.3)$ & \\
\hline$>7$ & $4(23.5)$ & $14(2.4)$ & \\
\hline PADUA score & $10.0 \pm 1.7$ & $8.4 \pm 1.5$ & $<0.001$ \\
\hline $6^{-7}$ & $1(5.9)$ & $191(33.2)$ & \\
\hline $8-9$ & $4(23.5)$ & $241(41.8)$ & \\
\hline $10-13$ & $12(70.6)$ & $144(25.0)$ & \\
\hline
\end{tabular}

Values are presented as mean \pm standard deviation or number (\%).

Group 1: PN to RN conversion group, group 2: PN group.

PN: partial nephrectomy, RN: radical nephrectomy, eGFR: estimated glomerular filtration rate, EBL: estimated blood loss, PADUA: preoperative aspects and dimensions used for an anatomical. 
13 cases were converted from robotic PN to robotic RN. There were many factors that influenced intraoperative PN conversion to RN. Tumor discrepancies were present in 8 of 17 conversion cases $(47.1 \%)$, invasion of hilar structure in 5 of 17 cases
(29.4\%), and failure to achieve clear tumor margin occurred in 2 of 17 cases $(11.8 \%)$. Difficulty in clamping multiple renal vessels and renal vessel injuries occurred in 1 of 17 (5.9\%) intraoperative PN to RN conversion cases, respectively (Table 2).

Table 2. The multiple reasons to intraoperative conversion to radical nephrectomy (RN)

\begin{tabular}{|c|c|c|}
\hline Reason & Scheduled operation-conversion type of $\mathrm{RN}$ & Number $(\%)$ \\
\hline \multirow[t]{4}{*}{ Tumor discrepancy } & & $8(47.1)$ \\
\hline & OPN-ORN & $2(11.8)$ \\
\hline & RPN-ORN & $0(0)$ \\
\hline & RPN-RRN & $6(35.3)$ \\
\hline \multirow[t]{4}{*}{ Invasion of hilar and collection structure } & & $5(29.4)$ \\
\hline & OPN-ORN & $1(5.9)$ \\
\hline & RPN-ORN & $0(0)$ \\
\hline & RPN-RRN & $4(23.5)$ \\
\hline \multirow[t]{4}{*}{ Achievement failure of clear margin } & & $2(11.8)$ \\
\hline & OPN-ORN & $1(5.9)$ \\
\hline & RPN-ORN & $0(0)$ \\
\hline & RPN-RRN & $1(5.9)$ \\
\hline \multirow[t]{4}{*}{ Difficulty in clamping due to multiple renal vessel } & & $1(5.9)$ \\
\hline & OPN-ORN & $0(0)$ \\
\hline & RPN-ORN & $0(0)$ \\
\hline & RPN-RRN & $1(5.9)$ \\
\hline \multirow[t]{4}{*}{ Renal vessel injuries } & & $1(5.9)$ \\
\hline & OPN-ORN & $0(0)$ \\
\hline & RPN-ORN & $0(0)$ \\
\hline & RPN-RRN & $1(5.9)$ \\
\hline
\end{tabular}

OPN: open radical nephrectomy, ORN: open radical nephrectomy.

Table 3. Univariate and multivariate analysis predicting conversion from PN to RN in patients with OPN and RPN

\begin{tabular}{|c|c|c|c|c|c|c|}
\hline \multirow{2}{*}{ Variable } & \multicolumn{3}{|c|}{ Univariate } & \multicolumn{3}{|c|}{ Multivariate } \\
\hline & OR & $95 \% \mathrm{CI}$ & p-value & OR & $95 \% \mathrm{CI}$ & p-value \\
\hline Age & 1.010 & $0.972-1.049$ & 0.620 & 1.016 & $0.976-1.059$ & 0.436 \\
\hline \multicolumn{7}{|l|}{ Exophytic rate } \\
\hline$\geq 50 \%$ & Reference & & & Reference & & \\
\hline$<50 \%$ & 2.751 & $0.933-8.110$ & 0.067 & 1.834 & $0.479-7.028$ & 0.376 \\
\hline \multicolumn{7}{|l|}{ Renal rim } \\
\hline Lateral & Reference & & & Reference & & \\
\hline Medial & 2.076 & $0.783-5.504$ & 0.142 & 1.113 & $0.370-3.346$ & 0.849 \\
\hline \multicolumn{7}{|l|}{ Renal sinus } \\
\hline Not involved & Reference & & & Reference & & \\
\hline Involved & 29.529 & $3.874-225.072$ & 0.001 & 9.075 & $1.010-81.508$ & 0.049 \\
\hline \multicolumn{7}{|c|}{ Urinary collecting system } \\
\hline Not involved & Reference & & & Reference & & \\
\hline Involved & 15.904 & $5.581-45.320$ & $<0.001$ & 3.944 & $1.152-13.506$ & 0.029 \\
\hline \multicolumn{7}{|l|}{ Tumor size $(\mathrm{cm})$} \\
\hline$<4$ & Reference & & & Reference & & \\
\hline$\geq 4$ & 1.029 & $1.012-1.046$ & 0.001 & 1.017 & $0.995-1.039$ & 0.132 \\
\hline
\end{tabular}

PN: partial nephrectomy, RN: radical nephrectomy, OPN: open radical nephrectomy, RPN: robotic radical nephrectomy, OR: odds ratio, CI: confidence interval. 


\section{Univariate Analysis for Predicting PN Conversion to RN}

We evaluated each item of the PADUA classification system to determine its impact on intraoperative PN to RN conversion rate (Table 3). According to the results of univariate analyses, tumor size $\geq 4 \mathrm{~cm}(\mathrm{OR}, 1.029 ; \mathrm{p}=0.001)$, UCS involvement $(\mathrm{OR}, 15.904 ; \mathrm{p} \leq 0.001)$ and renal sinus involvement $(\mathrm{OR}$, 29.529; $\mathrm{p}=0.001$ ) were strongly associated with conversion of PN to RN. The other factors constituting PADUA score system such as exophytic rate of tumor, tumor size, and laterality of renal rim were not associated with conversion of PN to RN (Table 3).

\section{Multivariate Analysis for Predicting PN Conversion to $\mathbf{R N}$}

After conducting multivariate analysis to adjust for possible confounding parameters, it showed that UCS involvement (OR, 3.944; $\mathrm{p}=0.029$ ) and renal sinus involvement (OR, 9.075; $\mathrm{p}=0.049$ ) were significantly associated with conversion of PN to RN. Tumor size $\geq 4 \mathrm{~cm}$, which was strongly related to PN conversion to $\mathrm{RN}$ in univariate analysis, failed to achieve independent predictor status in multivariate analysis (Table 3).

\section{DISCUSSION}

PN is the gold-standard treatment of small renal tumors with clinical stage $1 .{ }^{1}$ Van Poppel et al. ${ }^{15}$ previously contended that $\mathrm{RN}$ showed a better result than PN in decreasing perioperative complication, required a shorter operation time, and resulted in less morbidity and mortality. However their results were reported before more PN experience had emerged. Our team of urology surgeons has significantly more experience in PN in recent years, and we think that PN shows better results than $\mathrm{RN}$ for treating small renal tumors with respect to renal functional preservation. Some factors such as the volume of remaining renal parenchyma, and intact renal sinus or UCS require consideration. When minimally invasive PN encounters difficulties, we recommend conversion to OPN instead of $\mathrm{RN}^{15}$ Thus it is important to predict the likelihood of needing to intraoperatively convert from $\mathrm{PN}$ to $\mathrm{RN}$.

There were many factors that could affect the need for intraoperative conversion from $\mathrm{PN}$ to $\mathrm{RN}$. We divided these factors into anatomical aspects and pathophysiological aspects. In terms of anatomical aspects, large renal tumors or tumor dis- crepancies made dissection of the renal tumor more difficult and reduced the amount of remaining renal parenchyma. Additionally, involvement of renal sinus and UCS made it difficult to discern clear tumor margins or the complete renal pelvis, and resulted in an increased $\mathrm{PN}$ to $\mathrm{RN}$ conversion rate. We found that tumor involvement of renal sinus was the most significant factor of intraoperative conversion to $\mathrm{RN}$ with odd ratio of 9.075. UCS involvement also had a significant impact on radical conversion during PN. Although they were not counted in the evaluation, we believe additional intraoperative factors such as difficulty of vessel clamping, and renal vessel injuries might have influenced on radical conversion during PN in some degree. Thus, radical conversion was in most cases caused by anatomical factors.

In a study by Galvin et al., ${ }^{5}$ the conversion rate to RN was higher with larger renal tumors and when tumors were located closer to renal center. Long et al. ${ }^{16}$ observed that increased renal tumor size and higher RENAL nephrometry scores increased the intraoperative $\mathrm{PN}$ to $\mathrm{RN}$ conversion rate. However, their classification of renal tumors to the medial or peripheral kidney was ambiguous, and reproducibility was poor. Thus there remains no consensus regarding which factors, besides tumor size, increase the $\mathrm{PN}$ to $\mathrm{RN}$ conversion rate.

For evaluating the influence of any parameter on radical conversion rates in NSS, it is necessary that the reproducibility of diagnostic tools is high and assessment is easy. Because of these reasons, we investigated the effect each item of the PADUA classification scale on intraoperative conversion from PN to RN. The PADUA classification is composed of six parameters, plus anterior or posterior surface. Among these parameters, tumor size and the involvement of the renal sinus/UCS were the most important factors in predicting the need for radical conversion. Tumor location was not a predictor of conversion in our study, in contrast to findings in other studies. ${ }^{5}$ We showed that tumor involvement of UCS or renal sinus were the most important factors in predicting intraoperative PN to $\mathrm{RN}$ conversion, in both univariate and multivariate logistic regression analyses.

The pathophysiological condition of patients should be considered when selecting the operation method for NSS. If patients have advanced chronic kidney disease (stage $\geq 3$ ) along with a comorbidity such as uncontrolled diabetes, hypertension, coronary heart disease, or dyslipidemia, it is important that operation time and anesthesia time are minimized to enhance pa- 
tient outcomes. Galvin et al., ${ }^{5}$ in their survey found that the American Society of Anesthesiologists physical status classification grade, which is a 12 surrogate marker of comorbidity, did not influence the conversion rate of PN to RN. This suggests that PN conversion rates to RN were not affected by comorbidity itself, but instead by a lowered surgeon's threshold to radical conversion because it was worried to negatively affect the general condition of patients after surgery. If the operator becomes more experienced with $\mathrm{PN}$ and predicting the radical conversion rate before operating, unscheduled PN to RN conversions may be reduced. Patients with local symptoms like hematuria, flank pain, and fever were reported to have a higher PN to RN conversion rate. ${ }^{6}$ The association of local symptoms to the rate of conversion to $\mathrm{RN}$ from $\mathrm{PN}$ should be further investigated in future studies.

In our results, 17 of 593 total cases $(2.9 \%)$ underwent PN to RN conversion, with 4 cases being conversion from OPN to ORN. Our conversion rate to RN was remarkably different from those of Long et al. ${ }^{16}$ (16\% PN to RN conversion rate), and were also lower than the rate reported by Galvin et al. ${ }^{5}$ (6\% conversion rate). The reasons for our lower conversion rate may be that our procedure was performed by a highly experienced staff urologic surgeon in Seoul National University Bundang Hospital.

There were some limitations to our study. First, this study was done retrospectively, but these results are viable because the PADUA classification was only introduced in 2009. Second, we did not compare the difference between the results of OPN and RPN. We investigated the impact of PADUA classification in overall NSS. Third, there may be selection bias in the more complex cases. The da Vinci Robotic Surgical System (Intuitive Surgical Inc., Sunnyvale, CA, USA) offers 3-dimensional vision and easy feasibility to renorrhaphy, and is more suitable for complex cases of NSS than LPN. OPN and RPN were successfully performed for the high-complexity cases because of our surgeon's accumulated experience and our advanced technology. Thus, cases that underwent both RPN and OPN were enrolled in this study.

\section{CONCLUSIONS}

The PADUA classification is a useful tool to predict the likelihood of intraoperative PN to RN conversion. Tumor involvement of UCS or renal sinus appear to be the best predictors of radical conversion in patients undergoing PN. Further surgical experience is required to differentially validate PADUA predictive power of radical conversion in OPN versus RPN.

\section{REFERENCES}

1. Patard JJ, Shvarts O, Lam JS, Pantuck AJ, Kim HL, Ficarra $\mathrm{V}$, et al. Safety and efficacy of partial nephrectomy for all T1 tumors based on an international multicenter experience. J Urol 2004;171(6 Pt 1):2181-5.

2. Huang WC, Elkin EB, Levey AS, Jang TL, Russo P. Partial nephrectomy versus radical nephrectomy in patients with small renal tumors: is there a difference in mortality and cardiovascular outcomes? J Urol 2009;181:55-61.

3. Thompson RH, Boorjian SA, Lohse CM, Leibovich BC, Kwon ED, Cheville JC, et al. Radical nephrectomy for pT1a renal masses may be associated with decreased overall survival compared with partial nephrectomy. J Urol 2008;179:468-71.

4. Licht MR, Novick AC. Nephron sparing surgery for renal cell carcinoma. J Urol 1993;149:1-7.

5. Galvin DJ, Savage CJ, Adamy A, Kaag M, O'Brien MF, Kallingal $\mathrm{G}$, et al. Intraoperative conversion from partial to radical nephrectomy at a single institution from 2003 to 2008. J Urol 2011;185:1204-9.

6. Wang AJ, Bhayani SB. Robotic partial nephrectomy versus laparoscopic partial nephrectomy for renal cell carcinoma: single-surgeon analysis of $>100$ consecutive procedures. Urology 2009;73:306-10.

7. Long JA, Yakoubi R, Lee B, Guillotreau J, Autorino R, Laydner $\mathrm{H}$, et al. Robotic versus laparoscopic partial nephrectomy for complex tumors: comparison of perioperative outcomes. Eur Urol 2012;61:1257-62.

8. Kutikov A, Uzzo RG. The R.E.N.A.L. nephrometry score: a comprehensive standardized system for quantitating renal tumor size, location and depth. J Urol 2009;182:844-53.

9. Ficarra V, Novara G, Secco S, Macchi V, Porzionato A, De Caro R, et al. Preoperative aspects and dimensions used for an anatomical (PADUA) classification of renal tumours in patients who are candidates for nephron-sparing surgery. Eur Urol 2009;56:786-93.

10. Li L, Lau WL, Rhee CM, Harley K, Kovesdy CP, Sim JJ, et al. Risk of chronic kidney disease after cancer nephrectomy. Nat Rev Nephrol 2014;10:135-45.

11. Okhunov Z, Rais-Bahrami S, George AK, Waingankar N, Duty B, Montag S, et al. The comparison of three renal tumor scoring systems: C-Index, P.A.D.U.A., and R.E.N.A.L. nephrometry scores. J Endourol 2011;25:1921-4.

12. Hayn MH, Schwaab T, Underwood W, Kim HL. RENAL nephrometry score predicts surgical outcomes of laparoscopic partial nephrectomy. BJU Int 2011;108:876-81.

13. Ellison JS, Montgomery JS, Hafez KS, Miller DC, He C, 
Wolf JS Jr, et al. Association of RENAL nephrometry score with outcomes of minimally invasive partial nephrectomy. Int J Urol 2013;20:564-70.

14. Minervini A, Vittori G, Salvi M, Sebastianelli A, Tuccio A, Siena G, et al. Analysis of surgical complications of renal tumor enucleation with standardized instruments and external validation of PADUA classification. Ann Surg Oncol 2013; 20:1729-36.

15. Van Poppel H, Da Pozzo L, Albrecht W, Matveev V, Bono
A, Borkowski A, et al. A prospective, randomised EORTC intergroup phase 3 study comparing the oncologic outcome of elective nephron-sparing surgery and radical nephrectomy for low-stage renal cell carcinoma. Eur Urol 2011;59:543-52.

16. Long JA, Arnoux V, Fiard G, Autorino R, Descotes JL, Rambeaud JJ, et al. External validation of the RENAL nephrometry score in renal tumours treated by partial nephrectomy. BJU Int 2013;111:233-9. 